\title{
A Metastatic Lipid-Rich Carcinoma of the Mammary Gland in a Female Cat: Clinicopathological, Histopa- thological and Immunohistochemical Features
}

\author{
Adrian Florin $\mathrm{GAL}^{1 *}$, Cornel CĂTOI ${ }^{1}$, Marian TAULESCU ${ }^{1}$, Viorel MICLĂUȘ ${ }^{2}$, Andras NAGY ${ }^{1}$, Raluca \\ MARICA $^{1}$, Roxana POPA ${ }^{1}$, Flaviu TĂBĂRAN ${ }^{1}$ \\ ${ }^{1}$ Department of Pathologic Anatomy and Necropsy, Faculty of Veterinary Medicine, University of Agricultural \\ Sciences and Veterinary Medicine Cluj-Napoca, Calea Mănăştur 3-5, Cluj-Napoca, 400372, Romania \\ ${ }^{2}$ Department of Histology, Faculty of Veterinary Medicine, University of Agricultural Sciences and \\ Veterinary Medicine Cluj-Napoca, Calea Mănăștur 3-5, Cluj-Napoca, 400372, Romania \\ * corresponding author: adrian.gal@usamvcluj.ro
}

Bulletin UASVM Veterinary Medicine 74(2)/2017

Print ISSN 1843-5270; Electronic ISSN 1843-5378

doi:10.15835/buasvmcn-vm:0006

\begin{abstract}
Lipid-rich invasive human breast cancer is a rare enigmatic entity among special types of infiltrating duct carcinoma. Our paper reports a lipid-rich mammary carcinoma in a female cat with the gross, microscopic and immunohistochemical description of the tumor. A 13-year-old intact adult female, mixed-breed cat was presented by the owner to the Laboratory of Pathologic Anatomy from the Faculty of Veterinary Medicine ClujNapoca, Romania. A complete necropsy examination was performed in our laboratory. The tissue samples were collected and processed by paraffin technique for further histological, histochemical, immunofluorescence and immunohistochemical examination. During the necropsy examination, a subcutaneous mass was discovered on the chest. Several variably sized, well-demarcated neoplasms were noted in the right axillary lymph node, right thoracic wall, pleura, lungs, liver, spleen and kidney. Histologically, the cells frequently formed tubuloacinar structures. The morphology of the described tumor showed features of a poorly differentiated mammary carcinoma. Numerous tumoral cells were large and polygonal, with abundant cytoplasm that showed foam-like cytoplasm. The tumoral cells contained either multiple small or large and solitary vacuoles that pushed the nucleus to the periphery of the cell. Intracytoplasmic vacuoles of the neoplastic cells were positive for Oil-Red-0 and negatively with Periodic Acid-Schiff. As for immunofluorescence/immunohistochemistry, nonvacuolated and vacuolated neoplastic cells were positive for cytokeratin and negative for vimentin. Histochemical and immunohistochemical analysis support a diagnosis of lipid-rich mammary carcinoma. This is the second reliable record of a lipid-rich mammary carcinoma in female cat and the first one with internal metastases.
\end{abstract}

Keywords: female cat, lipid-rich carcinoma, mammary gland, Oil-Red-O.

\section{INTRODUCTION}

Lipid-rich invasive human breast cancer is a rare enigmatic entity among special types of infiltrating duct carcinoma. Only a few cases are reported in the literature (Wrba et al.,1988). Lipidrich carcinoma was first described in women by Aboumrad et al. (1963). Some authors thought that the lipid droplets were produced and secreted by the tumour cells instead of being the result of regressive degeneration because lipid droplets existed uniformly in metastasized lymph nodes and in tumor cells undergoing mitosis (Reis-Filho et al., 2003).

In the veterinary literature, lipid-rich carcinoma of the mammary gland has been reported in several dogs and one cat(Espinosa De Los Monteros et al., 2003; Kamstock et al., 2005). Kamstock and others (2005) described the first case of a lipid- 
rich mammary carcinoma in 1.5-year-old female cat.

Our paper reports the second lipid-rich mammary gland carcinoma in a female cat with the gross, microscopic and immunohistochemical description of the tumour. Nevertheless, we analyzed the first lipid-rich mammary carcinoma in female cat with internal metastases.

\section{MATERIALS AND METHODS}

In December 2010, a 13-year-old intact adult female, mixed-breed cat was presented by the owner to the Laboratory of Pathologic Anatomy from the Faculty of Veterinary Medicine Cluj-Napoca, Romania. The cat showed severe clinical signs of cancer progression and was humanely euthanized. A complete necropsy was performed in our laboratory. The female cat had a subcutaneous mass on the right side of the chest (i.e., right thoracic mammary gland). During the necropsy examination, several variably sized, welldemarcated neoplasms were noted in the right axillary lymph node, right thoracic wall, pleura, lungs, liver, spleen and kidney.

The tissue samples (from primary and secondary neoplasms) were fixed in 10\% neutral buffered formalin. For histopathologic study, the tissue samples were subsequently embedded in paraffin wax, sectioned at $4 \mu \mathrm{m}$ thicknesses and stained with hematoxylin and eosin.

Additional histochemical stains were performed (i.e., Oil-Red-O and Periodic Acid Schiff staining). Formalin-fixed tissues were stained with Oil-Red-O (Sigma, code 0-0625) according to the method described by Prophet et al. (1994) (i.e., appropriate for wax-embedded tissue sections). Periodic Acid-Schiff (PAS) stain was performed on 4- $\mu$ m-thick, formalin-fixed, wax-embedded, routinely processed tissue sections.

Immunohistochemical and immunofluorescence staining methods for vimentin (rabbit monoclonal anti-vimentin antibody, cat no MA1-39574, Thermo scientific), Ki-67 proliferation marker (mouse monoclonal antihuman Ki-67 antigen, clone MIB-1, ref M7240, Dako) and cytokeratin (mouse monoclonal anti-human cytokeratin AE1/AE3, cat no E006, Linaris), were performed on formalin-fixed, wax-embedded tissue sections (4- $\mu$ m-thickness). Steam antigen retrieval in citrate buffer (pH 6.0) was used. Commercially available antibodies tested (i.e., vimentin, Ki-67 proliferation marker, and cytokeratin) were diluted according to the manufacturers' instructions.

The labeled Labelled Streptavidin Biotin method (LSAB+system-HRP, K0679, Dako) was used for vimentin and Ki-67 nuclear proliferation marker detection. Peroxidase activity was visualized with diaminobenzidine chromogen as per routine protocol to obtain a brown end product. Ki-67 positive cells were counted by 2 observers. Positive and negative nuclei (minimum 1.000 neoplastic cells) in 6 representative highpower fields were counted. The proportion of positive neoplastic cells (i.e., percentage) was calculated. Positive and negative control slides were included in each experiment. Tissue analysis was performed using an Olympus system for image acquisition and analysis, respectively an Olympus BX51 microscope equipped with Olympus Cell B software.

A fluorescent labeled secondary antibody was used for the detection and visualization of the cytokeratin (Rhodamine labeled goat antimouse polyclonal antibody, Abcam code ab 67861). The nuclei were visualized after staining with red pseudocolor (DRAQ5 ${ }^{\circledR}$, catalog number 4084, Cell Signalling Technology, Massachusetts, USA) in accordance with manufacturers' protocols. Samples were analyzed and images were collected using a Zeiss LSM 710 Confocal Laser Scanning Microscopy (Oberkochen, Germany) equipped with Argon and $\mathrm{HeNe}$ laser mounted on an Axio Observer Z1 Inverted Microscope. Specific visualization of the structures performed using the $543 \mathrm{~nm}$ and $633 \mathrm{~nm}$ excitation laser lines to detect Rhodamine (emission filters 547-660 nm) and respectively Draq 5 (emission filters 676$757 \mathrm{~nm}$ ). All images were recorded using a Plan Apochromat $63 \times(1.4$; oil immersion, DIC 27) Zeiss objective. The beam pathway splitter used was MBS 458/543. The image combining, processing, and analysis was performed using the standard ZEN software package offered by the Zeiss system manufacturer.

The diagnosis criterion of lipid-rich carcinoma of the mammary gland was based on WHO classification in dogs and cats (Misdorp et al., 1999). The histological grade was accessed using the classification system proposed by Goldschmidt et al. (2011). 


\section{RESULTS AND DISCUSSION}

During the necropsy examination, a poorlydemarcated subcutaneous neoplasm was noted in the right thoracic mammary gland. This mass measured $4.7 \mathrm{~cm} \times 3.6 \mathrm{~cm}$ and weighed $14 \mathrm{~g}$. On gross examination, the neoplasm was brown to gray-white, compact on the cut surface and moderately firm. The tumor presented poorly defined borders, ulceration, and was fixed to the skin and underlying muscle tissue. The regional right axillary lymph node and afferent lymphatics were invaded by the tumor (numerous small nodules of 0.2 to $0.7 \mathrm{~cm}$ were noticed to the

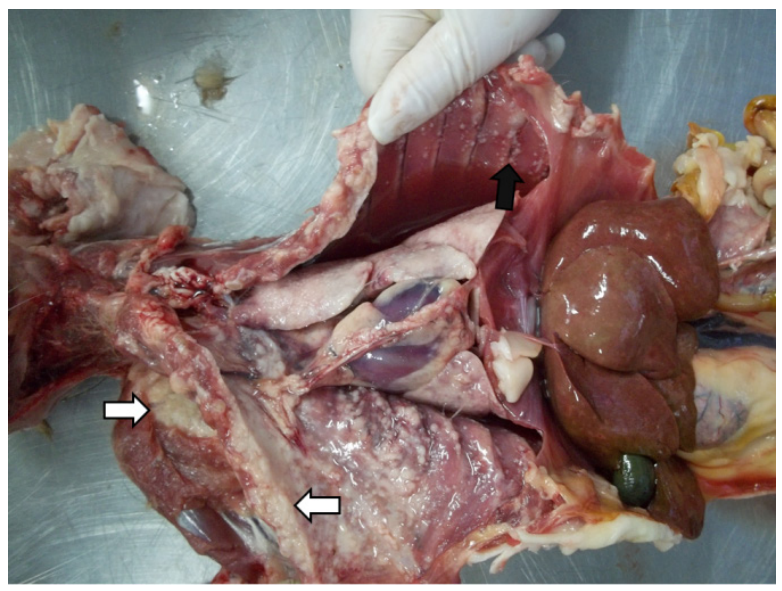

Fig. 1. Metastatic mammary carcinoma, histologic grade III, female cat. Tumor invasion throughout the right thoracic wall and spread in the parietal and visceral pleura (black arrow).

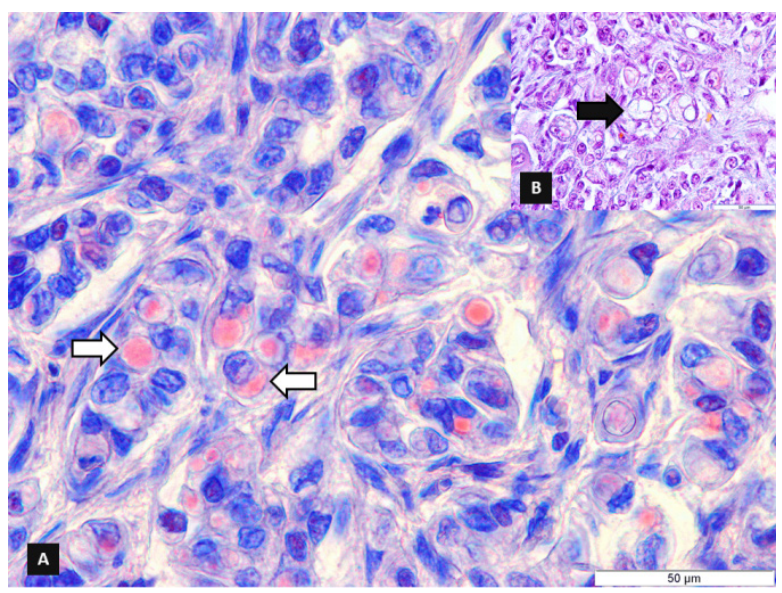

Fig. 3. Lipid-rich mammary carcinoma; female cat. The vacuoles of the tumor cells are Oil-Red-O positive (A), indicating the presence of lipids, and PAS negative (B). Mayer hematoxylin counterstain (A). medial side of the right member). The tumor invasion extended throughout the right thoracic wall and spread into parietal and visceral pleura (i.e., numerous masses of 0.2 to $1.2 \mathrm{~cm}$; Fig. 1). Additional tumoral masses were noted in the following organs: lungs (several masses of 0.5 to $3.3 \mathrm{~cm}$ ), right kidney (one mass of $0.5 \mathrm{~cm}$ ), liver (single neoplasm of $0.7 \mathrm{~cm}$ ) and spleen (masses of 0.3 to $1.0 \mathrm{~cm}$ ).

Histologically, the characteristic feature of the primary tumor was the presence of tubuloacinar structures. The neoplasm was only composed of epithelial cells (i.e., simple tumor). In most of the

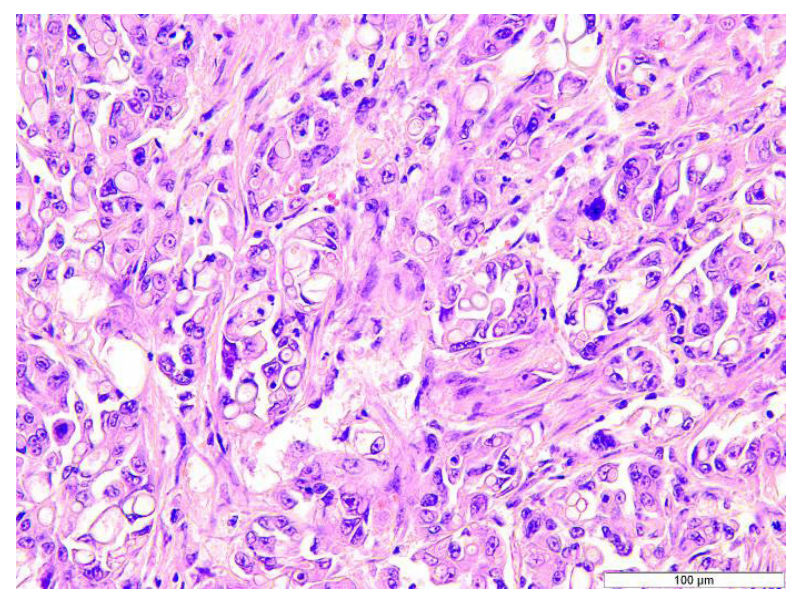

Fig. 2. Lipid-rich mammary carcinoma; female cat. Polygonal cells with foamy to monovacuolated cytoplasm. HE.

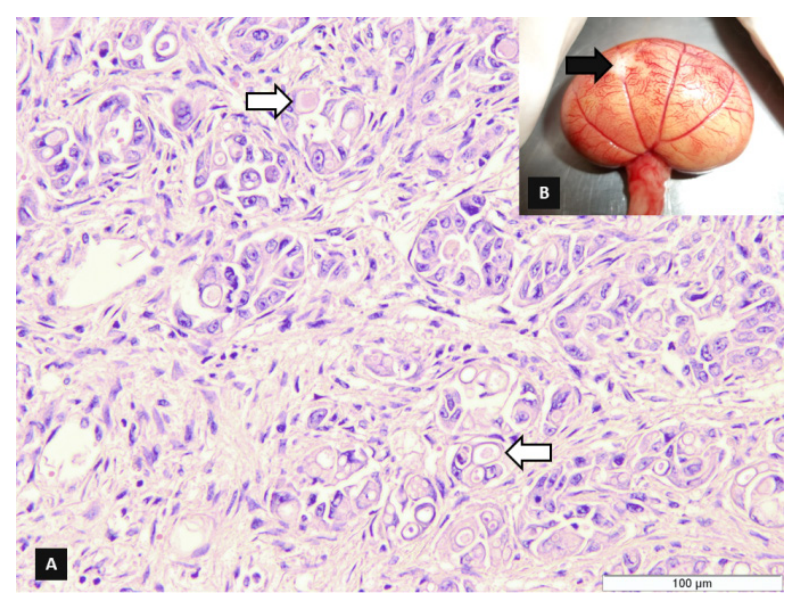

Fig. 4. Metastasis of a lipid-rich mammary carcinoma in the kidney; female cat. The presence of vacuoles in tumoral cells (A); gross aspect of the mass in the kidney (B). HE (A). 
microscopic fields the tubular structures had a solid aspect. These tubules consisted of multilayered epithelial cells forming solid nests. The cells were large and pleomorphic with a proportion of bizarre nuclei and a high mitotic count (Ki-67 proliferation marker percentage was $28.18 \%$ ). To the periphery of the tumor, individual cells and small clusters of cells were present. The histological findings were that of an infiltrating neoplasm composed of large, pleomorphic cells, often with large nuclei rich in chromatin and prominent nucleoli. Some cells were multinucleated showing anisokaryosis. The amount of stroma ranged from low to moderate. Nevertheless, the stroma contained a focal, lymphoplasmacytic infiltrate which did not intervene extensively between individual tumor cells. The border of the tumor was infiltrated by tumoral cells and vascular invasion was found. Morphology of the described tumor showed features of a poorly differentiated mammary carcinoma (i.e., histologic grade III).

Furthermore, numerous tumoral cells were large and polygonal, with abundant cytoplasm that showed foam-like or transparent cytoplasm. The tumoral cells contained either multiple and small or large and solitary vacuoles that pushed the nucleus to the periphery of the cell (signetring cells; Figs. 2-4). Intracytoplasmic vacuoles of the neoplastic cells were positive for Oil-Red-0 and neutral fat showed orange-red in cytoplasm (Fig. 3). The foamy or vacuolated cytoplasm of the neoplastic cells was unreactive with PAS staining (Fig. 3). As for immunohistochemistry/ immunofluorescence, nonvacuolated and vacuolated neoplastic cells were positive for cytokeratin (Fig. 5) and negative for vimentin. The secondary tumors (i.e., from the right axillary lymph node, right thoracic wall, pleura, lungs, liver, spleen and kidney) were composed predominantly of nonvacuolated neoplastic cells admixed with few vacuolated tumoral cells (Fig. 4).

Lipid-rich carcinoma of the breast is a rare variant, histologic phenotype of the human breast cancer, accounted for $<1 \%$ of all breast malignant tumors according to the World Health Organization (WHO) classification of breast tumors (Guan et al., 2011). As WHO classification defined, lipidrich carcinoma of the breast is a tumor showing conspicuous amounts of vacuolated or foamy lipid in the cytoplasm, which are strongly positive when stained for neutral fat, usually very highgrade nuclear features, and a poorly differentiated

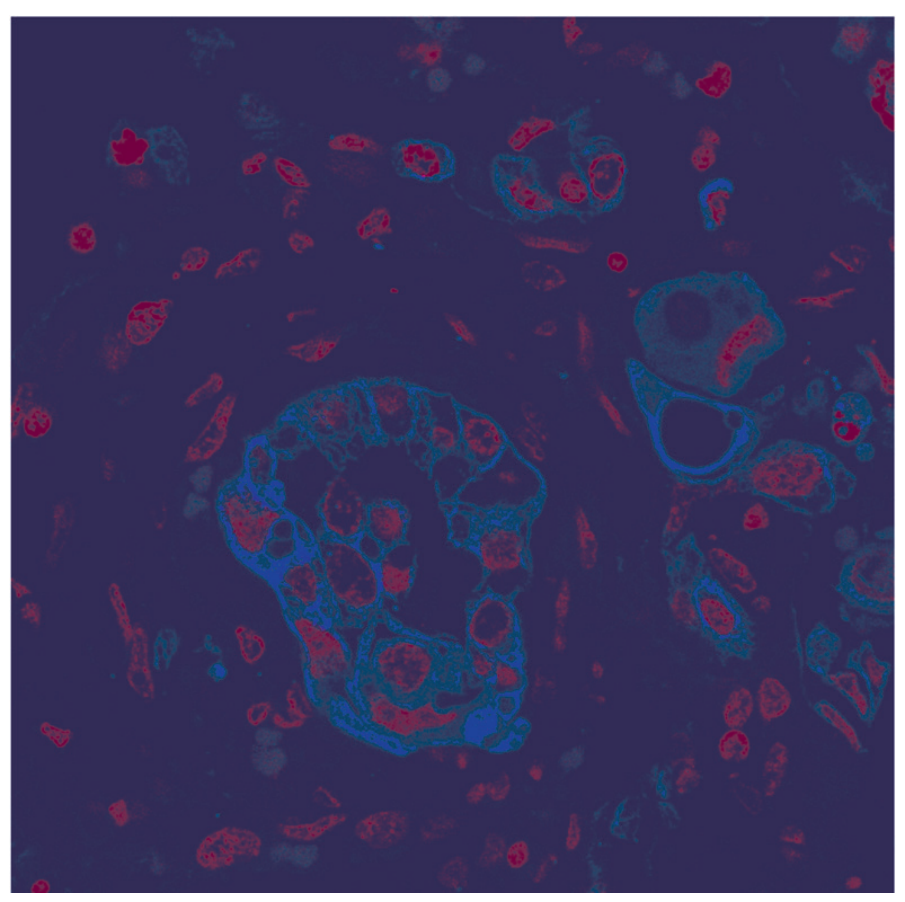

Fig. 5. Lipid-rich mammary carcinoma; cat. Tubuloacinar structure formed by polygonal cells with foamy to monovacuolated cytoplasm. Immunofluorescence, cytokeratin positive cells (blue) and red fluorescent nuclei (Draq 5); the image width is $134.6 \mu \mathrm{m}$. 
growth pattern, which means few/no glands or papillae (Guan et al., 2011).

In animals, the WHO tumor classification system indicates that lipid-rich carcinomas of the canine mammary gland are extremely uncommon (Misdorp et al., 1999). However, this tumor type was diagnosed only once in a female cat by Kamstock et al. (2005). The author described a 1.5-year-old female, intact, calico, outdoor, farm cat that had a single mass on the ventral abdomen. Six months after complete surgical excision of the mass, the female cat was clinically healthy with no signs of local recurrence or metastasis (Kamstock et al., 2005). Histologically, the cells frequently formed tubuloacinar structures. Individual cells contained moderate to abundant, foamy, eosinophilic cytoplasm with variably sized, distinct, round, clear vacuoles, which peripherally compressed the nucleus. Nuclei demonstrated marked atypia, were round to angular, variably sized, predominantly euchromatic, and basophilic with occasional prominent nucleoli. Mitotic figures were infrequent (Kamstock et al., 2005). Neoplastic cells demonstrated intense and diffuse immunoreactivity for cytokeratin and lacked immunoreactivity for vimentin. The vacuolar contents stained positively with Oil Red-O and negatively with periodic acid-Schiff and Alcian blue stains (Kamstock et al., 2005).

In our study, Oil Red-O staining positive and PAS staining negative suggested that neutral fat were placed into carcinoma cells cytoplasm. As for immunohistochemistry and immunofluorescence, the vacuolated neoplastic cells showed no reactivity to vimentin and were immunopositive for cytokeratin. The gross and histological findings in this study (i.e., marked cellular and nuclear atypia of the tumoral cells, large nuclei rich in chromatin, prominent nucleoli, multinucleated cells and anisokaryosis, and high mitotic index of $28.18 \%$ ) resemble to those reported in humans and dogs (Espinosa De Los Monteros et al., 2003; Guan et al., 2011). Human lipid-rich carcinoma has a more aggressive clinical course and worse prognosis than other types of breast cancer (Yoshimura et al., 2010). Furthermore, Espinosa De Los Monteros et al. (2003) reported approximate $71 \%$ of canine cases had evidence of metastasis. In the current case peritumoral neoplastic embolization and distant metastases in the right axillary lymph node, right thoracic wall, pleura, lungs, liver, sple- en and kidney were seen. In contrast to our study, no metastatic lesions were identified in the feline case reported by Kamstock et al. (2005).

In dogs, lipid-rich carcinoma has clinical features of inflammatory mammary carcinoma and is associated with recurrence and metastases (Yoshimura et al., 2010). Some recent studies described a number of cases of canine lipid-rich mammary carcinoma (Espinosa De Los Monteros et al., 2003; Pena et al., 2003). A glandular epithelial immunophenotype was observed in the majority of tumor cells. All tumors lacked both estrogen and progesterone receptors, and five out of seven tumors gave rise to local recurrence and proximal or distant metastases or both (Espinosa De Los Monteros et al., 2003).

Similarly, a lipid-rich carcinoma of the mammary gland was diagnosed in a female Djungarian hamster (Phodopus sungorus), which was kept as an indoor pet. Histologically, the primary neoplasm was composed of 2 different cell populations: nonvacuolated glandular neoplastic cells with moderate atypia, and vacuolated neoplastic cells with marked atypia (positive for OilRed-O and Nile-blue staining) (Yoshimura et al., 2010). Although mucin-producing carcinomas, glycogen rich clear cell carcinomas, and secretory carcinomas may contain cytoplasmic vacuoles in the neoplastic cells, they can be differentiated by staining reactions with PAS, and/or mucicarmine (Ellis et al., 2003).

\section{CONCLUSION}

In relation with the previously described morphological features, an unusual mammary carcinoma was described in a female cat. Histochemical and immunohistochemical analysis support a diagnosis of lipid-rich mammary carcinoma. It is the second reliable record of a lipid-rich mammary carcinoma in female cat and the first one with internal metastases. However, because of the high rarity of this neoplasm, many aspects of its pathology remain to be elucidated.

\section{REFERENCES}

1. Aboumrad MH, Horn RC, Fine G (1963). Lipid secreting mammary carcinoma. Report of a case associated with Paget's disease of the nipple. Cancer 16:521-525.

2. Ellis IO, Schnitt SJ, Sastre-Garau X, Bussolati G, Tavassoli FA, Eusebi V (2003). Invasive breast carcinoma. In Tavassoli FA, Devilee P, eds Pathology \& genetics: tumours of the breast and female genital organs. World Health Organization 
Classification of Tumours Series. IARC Press, Lyon, France, $13-59$.

3. Espinosa De Los Monteros A, Hellmen E, Ramirez GA, et al. (2003). Lipid-rich carcinomas of the mammary gland in seven dogs: clinicopathologic and immunohistochemical features. Vet. Pathol., 40:718-723.

4. Goldschmidt MH, Pena L, Rasotto R, Zappulli V (2011). Classification and grading of canine mammary tumors. Vet. Pathol., 48:117-131.

5. Guan B, Wang H, Cao S, et al. (2011). Lipid-rich carcinoma of the breast clinicopathologic analysis of 17 cases. Ann, Diagn, Pathol ,15:225-232.

6. Kamstock DA, Fredrickson R, Ehrhart EJ (2005). Lipidrich carcinoma of the mammary gland in a cat. Vet Pathol 42:360-362.

7. Misdorp W, Else RW, Hellmen E, Lipscomb TP (1999). Histological classification of the mammary tumors of the dog and cat. World Health Organization International Histological Classification of Tumors of Domestic Animals, 2nd series, vol. 7, Armed Forces Institute of Pathology and World Health Organization, Washington, DC.
8. Pena L, Dolores Perez-Alenza M, Rodriguez-Bertos A, Nieto A (2003). Canine inflammatory mammary carcinoma: histopathology, immunohistochemistry and clinical implications of 21 cases. Breast Cancer Res Tr 78:141-148.

9. Prophet EB, Mills B, Arrington JB, Sobin LH (1994). Laboratory methods in histotechnology, Armed Forces Institute of Pathology, American Registry of Pathology, Washington, DC, 178.

10. Reis-Filho JS, Fulford LG, Lakhani SR, Schmitt FC (2003). Pathologic quiz case: a 62 -year-old woman with a $4.5-\mathrm{cm}$ nodule in the right breast. Lipid-rich breast carcinoma. Arch Pathol Lab Med 127:396-398.

11. Wrba F, Ellinger A, Reiner G, Spona J, Holzner JH (1988). Ultrastructural and immunohistochemical characteristics of lipid-rich carcinoma of the breast. Virchows Arch A 413:381385.

12. Yoshimura H, Kimura N, Nakahira R, Michishita M, OhkusuTsukada K, Takahashi K (2010). Lipid-rich carcinoma in the mammary gland of a Djungarian hamster (Phodopus sungorus). J Vet Diagn Invest 22:305-309. 